Revue des patrimoines

Patrimoine en situation : l'Inventaire général entre histoire et prospective

\title{
L'architecte Pierre Le Mousseux (1687 - 1740) : ses dernières années en Bourgogne
}

\section{Brigitte Fromaget}

\section{(2) OpenEdition}

Journals

Édition électronique

URL : http://journals.openedition.org/insitu/9447

DOI : 10.4000/insitu.9447

ISSN : 1630-7305

Éditeur

Ministère de la culture

Référence électronique

Brigitte Fromaget, «L'architecte Pierre Le Mousseux (1687 - 1740) : ses dernières années en

Bourgogne », In Situ [En ligne], 6 | 2005, mis en ligne le 01 septembre 2005, consulté le 22 avril 2019.

URL : http://journals.openedition.org/insitu/9447 ; DOI : 10.4000/insitu.9447

Ce document a été généré automatiquement le 22 avril 2019

\section{cc) (†)}

In Situ Revues des patrimoines est mis à disposition selon les termes de la licence Creative Commons Attribution - Pas d'Utilisation Commerciale - Pas de Modification 4.0 International. 


\title{
L'architecte Pierre Le Mousseux (1687 - 1740) : ses dernières années en
} Bourgogne

\author{
Brigitte Fromaget
}

1 Lors de son séjour à Rennes, Le Mousseux ne se contente pas de collaborer à l'œuvre de Gabriel, il dresse également un certain nombre de plans d'édifices publics pour des projets qui n'ont pas abouti, avec, sur la demande de l'intendant, une révision «à la baisse » des travaux pour la place Neuve et l'hôtel de ville prévus par Jacques Gabriel ${ }^{1}$. Ce dernier a-t-il considéré que son adjoint outrepassait son rôle d'exécutant? Toujours est-il qu’à la suite de cette affaire il est remplacé par l'ingénieur Joseph Abeille en mai 1730.

Curieusement, en dépit des différends qui les ont opposés à Rennes en 1733, Gabriel fait venir Le Mousseux à Dijon où il le recommande expressément aux Élus Généraux, n'hésitant pas à vanter ses nombreuses qualités, non seulement pour la conduite et la surveillance du chantier du palais des États, mais aussi pour «toutes directions d'ouvrages, devis à dresser dont on le chargerait $»^{2}$. La Province ne se prive pas de l'employer pour divers travaux notamment comme ingénieur et expert aux Ponts et Chaussées ; c'est à ce titre qu'il intervient dans la reconstruction du pont de Seurre par Gabriel $^{3}$ et l'on peut juger de ses compétences en la matière à la lecture du rapport rédigé par ses soins sur l'effondrement du pont en cours de travaux, qu'il explique par la nature du terrain sur lequel on avait édifié les piles des arches.

3 Peu à peu, Pierre Le Mousseux prend une place grandissante auprès des Élus qui le sollicitent pour divers ouvrages et, comme à Rennes, il propose à plusieurs reprises des projets qui concurrencent ceux de l'architecte du roi, notamment pour la chapelle des Élus et le pont de Seurre. Toutes les activités qui lui sont demandées et les projets qu'il a dressés tendent à prouver qu'il est un architecte de talent et que ses compétences sont appréciées et reconnues. Le procès-verbal de réception des travaux du palais des États, le 28 avril 1738, montre que Jacques Gabriel a tout lieu d'être satisfait du travail de son adjoint. Pourtant Le Mousseux est remercié par le prince de Condé dans une lettre datée 
du 29 août 1739. Il est probable que l'estime que lui portent les Élus et son rôle prépondérant tant comme directeur des travaux du palais des États que comme ingénieur de la Province lui ont attiré l'inimitié de deux protégés du prince, Julien Bonnichon, ingénieur de la Province, et Claude Monseigneur, inspecteur des bâtiments des États. À la suite de ce renvoi, on a perdu sa trace et cru qu'il avait définitivement quitté Dijon en septembre de cette même année ${ }^{4}$.

Figure 1

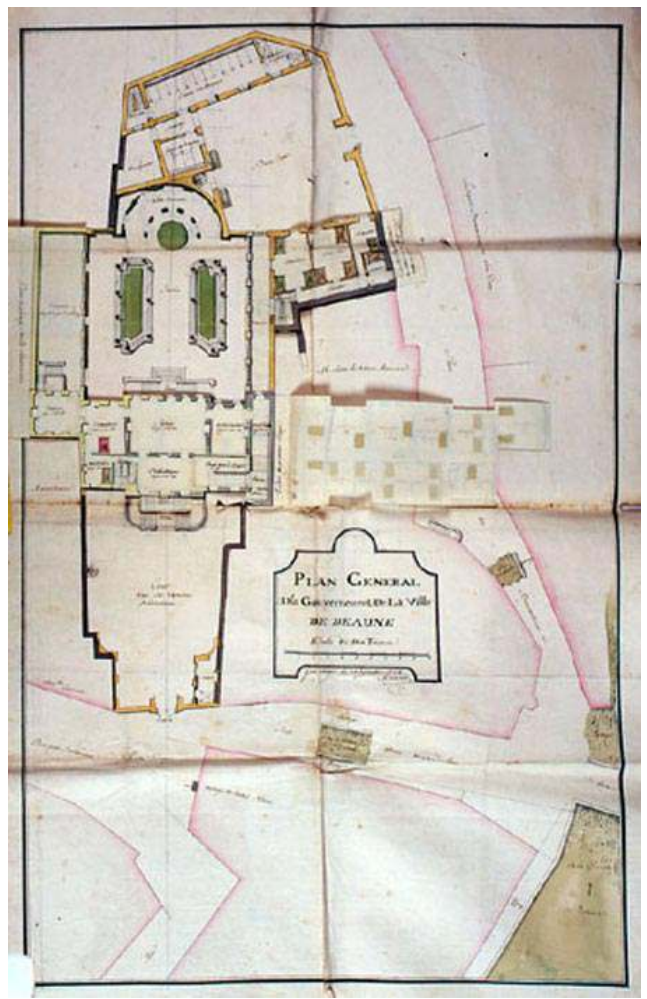

Projet d'aménagement pour loger le gouverneur de la ville de Beaune, 1738, plan de l'étage (Archives municipales de Beaune, carton 56, cote 30).

Repro. Inv. M. Thierry (C) Inventaire général, ADAGP, 1997.

Or des recherches d'archives effectuées par le service régional de l'Inventaire de Bourgogne ont permis de retrouver des documents relatifs à sa vie privée postérieurs à cette date, mais aussi trois projets inédits se rapportant à un hôtel particulier ${ }^{5}$ que la municipalité de Beaune devait acheter et transformer en logement pour le chevalier de Chavigny, nommé par le roi gouverneur militaire de la ville en 1737. Deux propositions sont présentées : l'une demandée par la Chambre de ville à son architecte, Denis Quinard, évite le plus possible un profond remaniement des bâtiments; la seconde, commandée par Chavigny, nettement plus ambitieuse, porte la mention « fait à Dijon le 20 septembre 1738 » et la signature « Mousseux » (fig. $\left.\mathbf{n}^{\circ} \mathbf{1}\right)$. Ce dernier essaie d'introduire une symétrie dans la forme irrégulière de la parcelle et prévoit des modifications plus importantes du logis, notamment la restructuration de la façade sur jardin et l'aménagement d'un grand salon au premier étage. Sont aussi prévues la reconstruction complète des communs et la création d'un jardin d'agrément à la française bordé de terrasses; il y dessine des parterres colorés et une allée couverte semi-circulaire pour fermer la perspective. L'année suivante, l'affaire tourne court, le gouverneur préférant obtenir de la ville une rente annuelle plutôt qu'un logement. 
5 Le deuxième projet, retrouvé dans le cadre de l'étude du patrimoine hospitalier régional, a trait à l'hôpital Saint-Laurent de Nuits-Saint-Georges ${ }^{6}$; il s'agit d'un devis " pour la construction de la nouvelle salle à faire à l'hôpital de Nuits et autres batimens suivant les plans, profils et élévations qui en ont été faits par le sieur Lemousseux le 25 may 1739 » (fig. $\left.n^{\circ} 2, n^{\circ} 3\right)$.

Figure 2

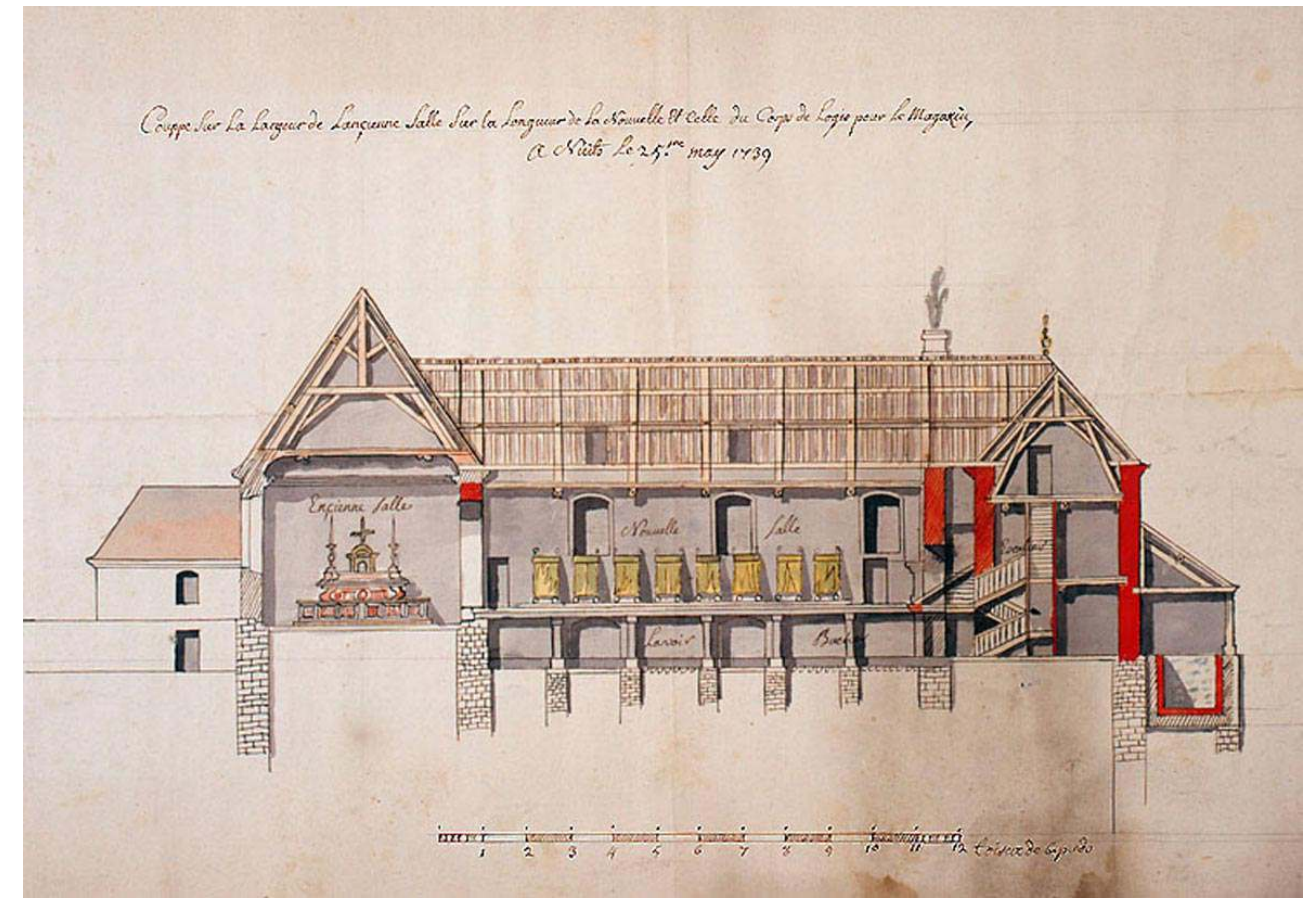

Coupe transversale sur la chapelle et coupe longitudinale de la nouvelle salle, 1739 (A. H. Nuits-SaintGeorges, carton 05).

Repro. Inv. M. Thierry (C) Inventaire général, ADAGP, 1997. 


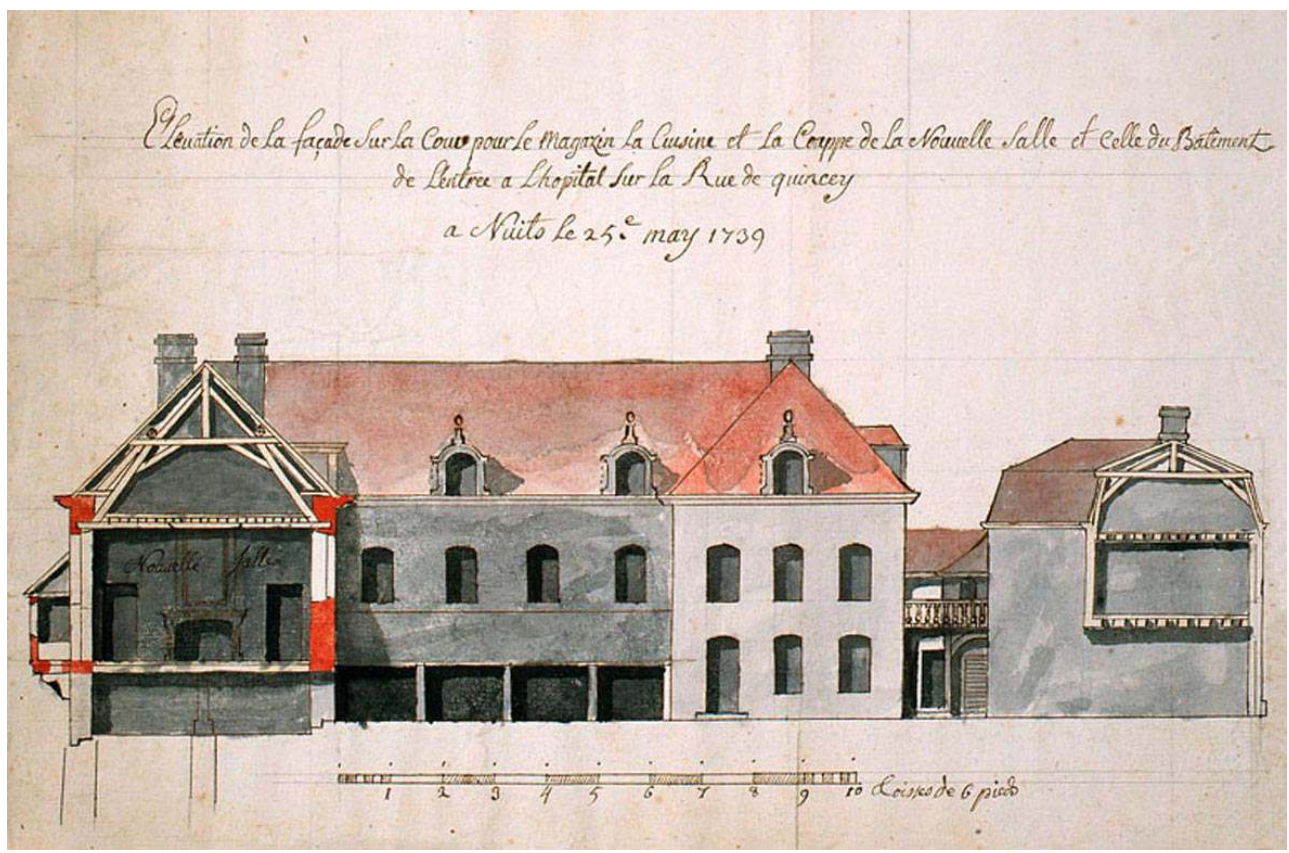

Élévation de la façade sur cour du bâtiment en retour d'équerre, 1739 (A. H. Nuits-Saint-Georges, carton 05)

Repro. Inv. M. Thierry (C) Inventaire général, ADAGP, 1997.

6 Outre une partie de ces documents graphiques, les archives de l'établissement possèdent encore des dessins de lambris décoratifs, très probablement de la main de Pierre Le Mousseux, dont on ne sait s'ils ont été réalisés (fig. $\left.\mathbf{n}^{\circ} 4\right)$. Ce n'était pas la première fois qu'il se préoccupait de décor intérieur, ayant déjà fourni et fait réaliser celui de la chambre des Élus à Dijon, en 1737. 


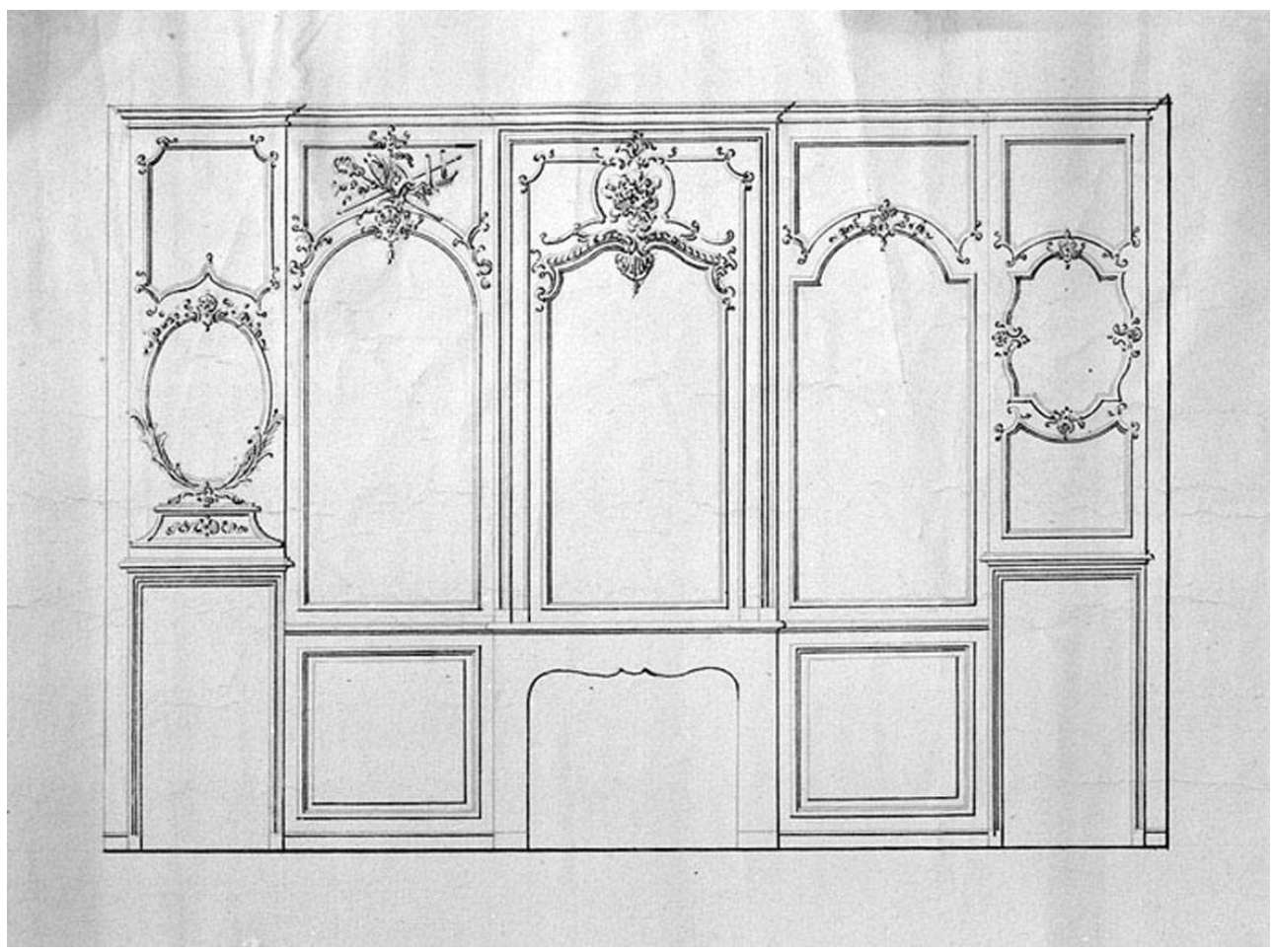

Projet de lambris, 1739 (A. H. Nuits-Saint-Georges, sans cote).

Repro. Inv. M. Thierry (C) Inventaire général, ADAGP, 1997.

7 À Nuits-Saint-Georges, il fait construire deux corps en équerre qui, venant s'appuyer sur le bâtiment déjà existant, donnent à l'ensemble un plan en U inspiré des grandes demeures comme beaucoup d'édifices hospitaliers de l'époque. Il ne néglige pas pour autant l'aspect pratique indispensable au bon fonctionnement de l'hôpital ; le bâtiment de la nouvelle salle des malades longe la rivière et abrite au niveau inférieur le lavoir et le bûcher.

Le dernier projet se rapporte aux travaux de réfection de la flèche de l'église abbatiale Saint-Bénigne de Dijon tombée lors du violent orage du 17 août 1738. Les religieux nomment Pierre Le Mousseux comme expert et c'est probablement à la demande de l'abbé que l'ingénieur du roi propose des plans, coupe et élévation, datés du 28 mai 1740, pour l'édification d'un nouveau clocher ${ }^{7}$. Celui-ci est largement inspiré de la version à l'économie du projet de Gabriel pour la tour de l'horloge de Rennes que Le Mousseux avait fait sur commande de l'intendant: on y retrouve des niveaux caractérisés par un décor d'architecture différent et de grandes ouvertures en plein-cintre pour l'un, en arc segmentaire pour l'autre, un fronton triangulaire interrompant un large bandeau mouluré, des pots à feu en amortissement et surtout l'insolite toiture en bulbe. Sans doute pour des raisons financières, la flèche sera reconstruite à l'identique, avec remploi des matériaux récupérables, par l'entrepreneur dijonnais Linassier.

Pour ce qui concerne sa vie en Bourgogne après sa disgrâce en 1739, l'indice qui a permis d'en esquisser le parcours provient d'une délibération de la mairie de Beaune : en 1742, Jeanne du Russeau sa veuve, demeurant à Dijon, réclame le paiement des plans du logement du gouverneur dressés par son mari ${ }^{8}$. À partir de ces indications, des recherches 
ont été entreprises dans les registres paroissiaux et les archives notariales pour reconstituer les dernières années de sa vie.

Sa date de naissance est donnée par «l'extrait baptistaire du 8 mars $1687 »^{9}$, retrouvé chez lui lors de l'inventaire après décès, les autres documents se rapportant à des événements plus récents ${ }^{10}$.

11 Le 3 mai 1736, «Pierre Mousseux ingénieur du Roy », fils de Pierre Mousseux, architecte et entrepreneur des bâtiments du roi à Fontainebleau, épouse Jeanne du Russeau, fille et veuve de conseillers du roi maîtres des comptes à Dole ${ }^{11}$. On compte parmi les témoins François Benoist Roche, avocat et échevin de cette ville, Étienne Rossignol, trésorier des mortes payes en Bourgogne, Jean Joly, chanoine régulier et Michel Potier, prud'homme de la ville, et l'inspecteur des ouvrages publics, Claude Monseigneur, un de ses rivaux qui ne sera pas totalement étranger à son renvoi par le prince de Condé à peine trois ans plus tard.

12 Le contrat de mariage stipule que le marié apporte la somme de 15000 livres provenant notamment d'une rente de 300 livres sur les haras du roi en Normandie; la dot de sa femme se monte à 44800 livres $^{12}$.

Le 23 janvier 1737 et le 25 janvier 1738, naissent Jeanne Catherine et Catherine Rose, baptisées à Saint-Médard et, le $1^{\text {er }}$ février 1739, Pierre, baptisé à Saint-Philibert ${ }^{13}$.

Le 18 avril 1740, il achète un domaine situé à Saint-Seine-sur-Vingeanne ${ }^{14}$, moins de six mois avant de mourir de maladie à Seurre, âgé de 53 ans. L'acte de décès du 9 octobre 1740 le cite comme ingénieur du Roi et comme l'un des entrepreneurs du pont de Seurre ${ }^{15}$ . Il est enterré le lendemain à l'église Saint-Martin de Seurre. On constate non seulement qu'il n'a pas quitté Dijon en septembre 1739, mais qu'il a continué de s'occuper de la reconstruction du pont de Seurre d'après les plans et devis de Jacques Gabriel.

Après sa mort de nombreux créanciers se présentent et parmi eux, Claude Rousselot, avocat au Parlement, conseiller du roi et contrôleur au grenier à sel de Seurre. Il réclame deux cent trente et une livres pour avoir payé le chirurgien qui a soigné l'architecte et pour le coût des obsèques. Il expose également que «le sieur Lemousseux s'étant mis en pension chez luy avec son valet pour veiller à la construction et entreprise du pon de Seurre étant mort chez luy, il lui est deu la somme de trois cents livres tant pour les embaras et préjudices extraordinaires que la mort dudit Sr Lemousseux luy ont causés $\ldots »^{16}$.

16 Ses dettes sont si importantes que sa veuve doit demander à la ville de Dijon une pension pour élever ses enfants ${ }^{17}$.

17 L'inventaire après décès en sa maison rue du Chapeau rouge à Dijon donne une certaine idée de son train de vie: abondance de linge de maison, d'ustensiles de cuisine, de mobilier, de vêtements de luxe parfois usés, de tapisseries (verdures formant tentures de chambre pour la plupart), livres reliés en veau et nombreux tableaux dont deux portraits de Le Mousseux et un de son épouse. Sont également notés les objets en rapport avec son métier: des crayons, de l'encre de chine, une boussole, des instruments de mathématiques, un plan de Dijon, une carte de la Bourgogne, une grande quantité de plans et dessins parmi lesquels le logis du roi et le palais des États, « un grand livre en brochure italien contenant le dessin de l'amphithéâtre de Flavius et un grand livre in folio relié en veau contenant les dix livres d'architecture de Vitruve ». Le chapitre «titres et papiers " contient des documents faisant allusion à des activités professionnelles non connues : un marché passé en 1729 avec deux menuisiers de Rennes pour des ouvrages à 
faire chez le marquis de Liré, et deux autres pour des travaux de plomberie et de charpente pour le nouveau clocher de l'église Saint-Bénigne de Dijon.

Le résultat de cette recherche apporte un nouvel éclairage sur le destin d'un praticien qui mérite mieux que sa réputation posthume de simple exécutant. Il était conscient de son talent, que le premier architecte du roi lui-même avait reconnu en lui confiant la direction de ses chantiers à Rennes puis à Dijon. Il tenta de sortir de l'ombre de Jacques Gabriel en exerçant, parallèlement aux grands travaux de la Province, ses propres activités en matière d'architecture et de décoration intérieure. Il est probable que d'autres aspects de son œuvre sont encore à découvrir et plus particulièrement en Bourgogne où il avait fondé une famille et projetait sans doute de mener une carrière à son compte, interrompue par une mort prématurée.

\section{NOTES}

1. NIERES, Claude. La reconstruction d'une ville au XVIIIe siècle - Rennes, 1720-1760. Paris : Klincksieck, 1972. BERGOT, François. L'hôtel de ville de Rennes. Les Gabriel. Paris : Picard, 1982, p. 56-71.

2. BEAUVALOT, Yves. Jacques Gabriel à Dijon. Les Cahiers du Vieux Dijon, $\mathrm{n}^{\circ} 8$ à 11 (1983) ; Gabriel à Dijon. Les Gabriel. Paris : Picard, 1982, p. 85 à 95. Recherches sur le décor du Palais des États à Dijon aux XVIIe et XVIIIe siècles. Mémoires de la Commission des Antiquités de la Côte d'Or, t. XXXII (1980-1981), p. 415-443.

3. BEAUVALOT, Yves. Jacques Gabriel et la reconstruction du pont de Seurre (1722-1740). Mémoires de la Commission des Antiquités de la Côte d'Or.t. XXXIII (1982-1983), p. 311-383.

4. BEAUVALOT, Yves. Jacques Gabriel à Dijon. Les Cahiers du Vieux Dijon, nº 8 à 11 (1983), p. 104 et 151 note 145 .

5. FROMAGET, Brigitte. L'histoire de l'hôtel de Montille, actuelle sous-préfecture. Centre Beaunois d'Études Historiques, recueil n 18, mémoires tome LXXX (2000), p. 67 à 82. Voir dans la base Mérimée : http://www.culture.gouv.fr/documentation/merimee/accueil.htm Notice réf. IA21000092.

6. Voir dans la base Mérimée: http://www.culture.gouv.fr/documentation/merimee/ accueil.htm Notice réf. IA21000094.

7. A. D. Côte d'Or, $1 \mathrm{H} 134$ : Réparations à la flèche et aux bâtiments endommagés par la chute de ladite flèche le 17 août 1738. 1H 135 : Plans et élévation par Mousseux (1740). Le dessin de l'élévation du clocher a été présenté récemment à l'occasion d'une exposition du service éducatif des Archives départementales de la Côte-d'Or « Abbayes en Côte d'Or. Piété et pouvoir ».

8. A. M. Beaune : Registres des délibérations de la mairie 1738-1743, fo 243.

9. Le Mousseux est probablement né à Fontainebleau où son père est cité comme architecte entrepreneur des bâtiments du roi dans le contrat de mariage, et son frère Louis Gabriel comme entrepreneur de bâtiments dans l'inventaire après décès.

10. A. D. Côte d'Or, $B^{2} 356$ / $67: 5$ décembre 1740, Inventaires des meubles et effets, titres et papiers délaissés par le Sr Pierre Lemousseux Ingénieur demeurant en cette ville ; 2 au 7 janvier 1741 , vente des meubles et effets de la succession du sieur Pierre Lemousseux ingénieur pour le roi demeurant à Dijon; réclamation de Claude Rousselot et diverses pièces justificatives de créanciers. 
11. A. M. Dijon, B 577 : acte de mariage.

12. A. D. Côte d'Or, 4E 2 / 1399, notaire Cazotte père, année 1736 : contrat de mariage le 25 avril 1736 entre Mr Pierre Mousseux et dame Jeanne du Russeau.

13. A. M. Dijon, B 578 à 580 : Registres des paroisses Saint-Médard et Saint-Philibert.

14. A. D. Côte d'Or, 4E 12 / 8, notaire Béguillet : 18 avril 1740 : Ratification de la délivrance d'un domaine situé à Saint Seyne, Latour sur Vingeanne par le sieur Valentin d'Aubremont cavalier au régiment d'Anjou au profit du Sr Pierre Mousseux ingénieur du roi à Dijon. Saint-Seine-surVingeanne se trouve à une quarantaine de $\mathrm{km}$ au nord-est de Dijon et environ soixante-dix de Seurre.

15. A. D. Côte d'Or, 2E 607 / 3 : Seurre, acte de décès du 9 octobre 1740.

16. A. D. Côte d'Or, $B^{2} 356$ / $67: 5$ décembre 1740, Inventaires des meubles et effets, titres et papiers délaissés par le Sr Pierre Lemousseux Ingénieur demeurant en cette ville ; 2 au 7 janvier 1741, vente des meubles et effets de la succession du sieur Pierre Lemousseux ingénieur pour le roi demeurant à Dijon; réclamation de Claude Rousselot et diverses pièces justificatives de créanciers.

17. A. D. Côte d'Or, C 3403 et C 3468.

\section{RÉSUMÉS}

Considéré avant tout comme un architecte d'exécution, Pierre Le Mousseux n'a fait l'objet d'aucune étude particulière. Sa vie et son œuvre ne sont connues que pendant ses périodes de collaboration avec les architectes du roi : de1716 à 1724, il est en Normandie où il bâtit pour le roi le haras du Pin sous la direction de Robert de Cotte, de 1725 à 1730, il est à Rennes car Jacques Gabriel l'a choisi comme adjoint pour la reconstruction de la ville et, en 1733, il arrive à Dijon où l'architecte du roi fait de nouveau appel à lui pour le seconder dans l'édification de l'escalier et d'une aile du palais des États.

Seen primarily as an "execution" architect, Pierre Le Mousseux has never been studied as an individual creator. His life and his work are documented primarily during the periods when he collaborated with the King's architects: from 1716 to 1724 when he was in Normandy and built the royal stud farm of Le Pin, under the direction of Robert de Cotte; and from 1725 to 1730 when he was at Rennes, under the direction of Jacques Gabriel for the reconstruction of the city. In 1733 he came to Dijon where the King's architect again called on his assistance for the construction of a staircase and new wing of the States Palace

\section{INDEX}

Mots-clés : Pierre Le Mousseux, architecte, ingénieur, gouverneur, hôtel de Montille, souspréfecture de Beaune, hôpital, Nuits-Saint-Georges, Seurre, pont, Gabriel, Bourgogne, Rennes, architecture publique, ponts-et-chaussées, Julien Bonnichon, Claude Monseigneur, Chavigny, Denis Quinard, Saint-Bénigne, Dijon, Linassier, inventaire après décès

Keywords : stud farm, engineer 
AUTEUR

BRIGITTE FROMAGET

Ingénieur d'études. Inventaire. Région Bourgogne. brigitte.fromaget@culture.gouv.fr 